\title{
Rollback and Forking Detection for Trusted Execution Environments using Lightweight Collective Memory
}

\author{
Marcus Brandenburger \\ IBM Research - Zurich \\ burezurich.ibm.com \\ Matthias Lorenz \\ TU Braunschweig \\ mlorenzeibr.cs.tu-bs.de
}

\author{
Christian Cachin \\ IBM Research - Zurich \\ cca@zurich.ibm.com \\ Rüdiger Kapitza \\ TU Braunschweig \\ rrkapitzeibr.cs.tu-bs.de
}

June 20, 2017

\begin{abstract}
Novel hardware-aided trusted execution environments, as provided by Intel's Software Guard Extensions (SGX), enable to execute applications in a secure context that enforces confidentiality and integrity of the application state even when the host system is misbehaving. While this paves the way towards secure and trustworthy cloud computing, essential system support to protect persistent application state against rollback and forking attacks is missing.

In this paper we present $L C M$ - a lightweight protocol to establish a collective memory amongst all clients of a remote application to detect integrity and consistency violations. LCM enables the detection of rollback attacks against the remote application, enforces the consistency notion of forklinearizability and notifies clients about operation stability. The protocol exploits the trusted execution environment, complements it with simple client-side operations, and maintains only small, constant storage at the clients. This simplifies the solution compared to previous approaches, where the clients had to verify all operations initiated by other clients. We have implemented LCM and demonstrated its advantages with a key-value store application. The evaluation shows that it introduces low network and computation overhead; in particular, a LCM-protected key-value store achieves $0.72 \mathrm{x}-0.98 \mathrm{x}$ of a SGX-secured key-value store throughput.
\end{abstract}

\section{Introduction}

Despite numerous efforts by industry and academia cloud computing suffers still from trust issues [24, 33]. This is not surprising as companies possess limited control once their applications and data enter the cloud. Users have to trust the operating personal and a complex software stack composed of management software, virtualization layers, as well as commodity operating systems. On top, cloud providers are typically reluctant to share their exact system details because this information is critical for their business.

The recently released Software Guard Extensions (SGX) [31] technology of Intel is expected to make a change, as it addresses trust issues that customer face when outsourcing services to off-site locations and still gives cloud providers the freedom to not disclose their system details. SGX offers an instruction set extension that allows to establish trusted execution contexts, called enclaves. These enclaves might be tailored and comprise only a small dedicated fraction of an application [21, 8] or 
can contain an entire legacy application and the necessary operating system support [4, 2]. Thereby, the plaintext of enclave-protected data and code is only available for computation inside the CPU, and encrypted as soon as it leaves the CPU package again. In this way, enclave-residing data is even guarded against unauthorized accesses by higher privileged code and from attackers with administrative rights and physical access.

While SGX can be considered as a big step forward towards trustworthy cloud computing, some attack vectors nevertheless remain. One important open issue are rollback and forking attacks on stateful applications that make use of persistent storage. Whereas SGX provides mechanisms against mainmemory replay attacks, persistent storage is not under the direct control of SGX and therefore harder to secure. The need to handle system restarts, operating system crashes, and power outages makes a completely secure solution for state continuity difficult to achieve. Baumann et al. [4] who pioneered the field by proposing application enclaves acknowledge this issue and suggest to use a central external service that is contacted on every request. However, this only delegates the problem to an external entity, demands additional remote communication and adds another single point of failure. Strackx and Piessens [38], on the other hand, proposed abstractions on top of hardware-based trusted counters. This and similar approaches [14, 26, 32, 38] enable immediate detection of forking attacks but suffer from bad performance, as writing and reading trusted non-volatile counters for every request is timeconsuming. Finally, there are a number of approaches that do not rely on secure execution contexts, such as enclaves, but utilize only plain resources of an untrusted provider [7, 10, 11, 17, 30, 35]. These systems typically require cooperating clients to verify each server response. In particular, this comes with additional communication overhead between clients and server, and requires costly cryptographic verification.

In this paper we present Lightweight Collective Memory (LCM) - a distributed protocol to establish a collective memory amongst all clients of a remote application to detect integrity and consistency violations. By leveraging trusted execution environments (TEEs), such as SGX, LCM keeps client interaction and service state confidential. It ensures fork-linearizability [30], which denotes the strongest consistency notion among the clients that can be achieved in the presence of rollback attacks without direct client-to-client communication and in absence of trusted non-volatile memory. Furthermore, LCM notifies clients about operation stability. This criteria refers to stable operations where a client can be sure that its request has been acknowledged by a designated number of other clients. A typical size would be the majority of clients. Finally, compared to previous approaches that rely on trusted counters, applications secured by LCM can be migrated across physical TEEs and maintain their capability to detect rollback attacks and to enforce fork-linearizability.

We implemented LCM as a Java and C++ framework and demonstrate its advantage by securing a key-value store. We evaluated the performance of the prototype by using the YCSB benchmark and compare with native execution and SGX-secured approaches. It turns out that a SGX-secured key-value store achieves $0.42 \mathrm{x}-0.78 \mathrm{x}$ performance of unprotected native execution. However, the performance of LCM is $0.72 \mathrm{x}-0.98 \mathrm{x}$ of the SGX-secured key-value store throughput, while on top enabling rollback and forking detection.

The remainder of the paper is structured as follows. Sec. 2 provides a detailed problem description and outlines the necessary background. In Sec. 3, past solutions for state continuity are discussed and the goals of LCM are stated. Next, the overall architecture and the LCM protocol are introduced in Sec. 4. Sec. 5 provides the details of our implementation using SGX. Subsequently, Sec. 6 explains the evaluation results. Finally, Sec.7 7 outlines related approaches while Sec. 8 concludes the paper. 


\section{Problem description}

\subsection{System model}

We consider an asynchronous distributed system with $n$ clients $C_{1}, \ldots, C_{n}$ and a server $S$. The server contains a trusted execution environment (TEE), which hosts a trusted execution context $T$; this is an isolated, protected container that runs an application protocol and is trusted by the clients. A protocol $P$ specifies the behavior of the clients, the server $S$, and the trusted execution context $T$. All clients are correct, follow $P$, and mutually trust each other; clients and the server may crash but are able to recover with the help of stable storage, which they can access through load and store operations. In contrast, $T$ is correct but runs under the control of $S$ as explained in detail later; $T$ does not have direct access to stable storage and may lose its state. The server is either correct and follows $P$ or is Byzantine, deviating arbitrarily from $P$.

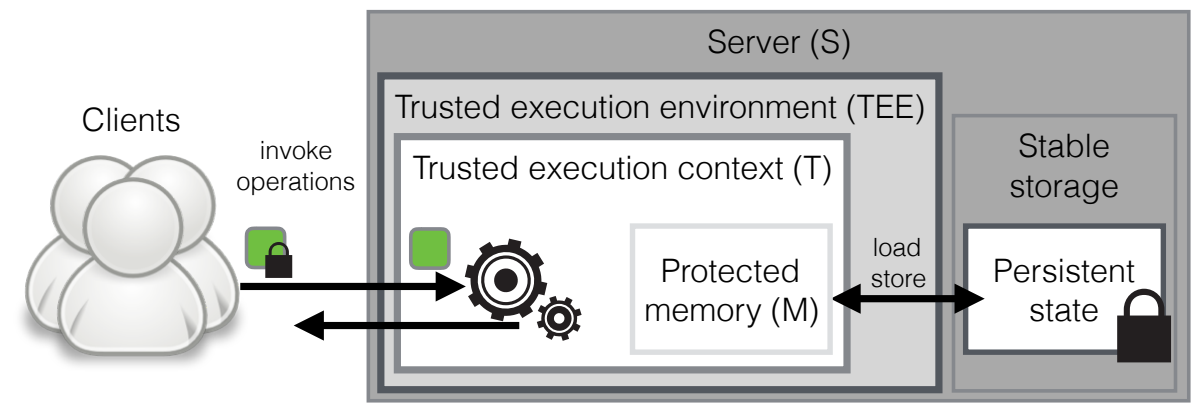

Figure 1. System model comprising trusted clients, a potentially misbehaving server $S$ that hosts a trusted execution context $T$.

The clients and $T$ interact by exchanging messages as specified by $P$. They communicate indirectly through the server which should forward messages among them. If $S$ is correct, then their communication is reliable and respects first-in first-out (FIFO) semantics; otherwise, $S$ may arbitrarily interfere with their messages. Clients have limited communication capabilities beyond this and do not interact with each other normally. The clients invoke a stateful application functionality $F$, which provides a set of operations; $F$ defines a response and a state change for every operation. The operations are executed by $T$ inside the TEE and, therefore, the state of $F$ is protected from a potentially malicious $S$. We use the standard notions of executions, histories, sequential histories, real-time order, concurrency, and well-formed executions from the distributed-computing literature [3]. In particular, every operation execution is represented by an invocation event and a response event. An operation is called complete when a client receives a response event. Two operations are concurrent if the invocation event of one of them occurs before the other operation is complete.

\subsection{Trusted execution context}

A TEE provides a secure context for executing applications, isolated from the server that hosts the TEE. It protects the confidentiality and integrity of code and data for the application running inside the execution context. More specifically, a trusted execution context $T$ is instantiated with a protocol $P$, which defines the program code executed by $T$. After server $S$ has created some $T, S$ may start, terminate, and restart $T$ at its discretion. Once $T$ has been created, $P$ running within $T$ cannot be modified anymore nor may any other protocol $P^{\prime}$ be executed in $T$. The server may also create and run multiple instances of $T$ concurrently. The time between instantiation and termination of $T$ is called an epoch. The entire lifetime of a trusted execution context can span multiple epochs. 
The TEE provides access to a secure random number generator that allows to build cryptographic primitives, such as key generation, encryption and digital signatures. The TEE operates a cryptographic key-management infrastructure rooted in a secret key protected by the TEE, which may provide a program-specific key to a trusted execution context. That is, a function get-key ${ }_{T, P}$ is available to $T$ when it executes protocol $P$ and returns a secret key $k$ that is specific to $P$ and the TEE. Another $T^{\prime}$, which is also instantiated with $P$, obtains the same $k$, but any $T$ running $P^{\prime} \neq P$ or any other TEE obtains a key different from $k$.

The clients can verify that a trusted execution context has been instantiated with a certain protocol $P$ and that $P$ is indeed running inside the TEE. This is essential for the assumption that $T$ is trusted. For this purpose clients leverage a procedure called remote attestation [1]. In short, a client with prior information about $P$ sends a challenge to $T$ and in return receives a cryptographic proof $\phi$ that reflects $P$ and the underlying TEE. The client then verifies $\phi$ and becomes convinced that $T$ runs $P$, based on the cryptographic protocol and on its trust in the TEE.

Furthermore, $T$ is equipped with a small protected memory area $M$ that can only be accessed by $T$. It holds the execution-specific state as defined by $P$. Neither the server nor any other trusted execution context can access or modify $M$. The protected memory is volatile, thus $M$ is only accessible within an epoch of $T$. In other words, when $T$ stops, crashes, or restarts, then $M$ is lost. This is not an issue for stateless protocols, but services without state are generally not very useful; in realistic applications, where the server maintains some state, $M$ must be restored after $T$ has been restarted. For this reason $M$ is stored externally on stable storage using load and store, so that $T$ can access state from another epoch.

\subsection{Threats}

Normally server $S$ is correct, but it may become malicious and behave incorrectly, when corrupted by an attacker or affected by a software bug. A malicious server has full control over the operating system, applications, and the data residing in memory and stable storage, but it cannot tamper with code and data in the trusted execution context. This means $T$ is correct and follows $P$ even though $S$ is malicious.

However, $S$ controls every interaction of $T$ with the environment. A malicious server may intercept, modify, reorder, discard, or replay messages to and from $T$. Although some of those attacks can be prevented by establishing a secure channel between a client and $T$, a malicious $S$ may simply discard their messages; such a denial-of-service (DoS) attack is outside the scope of this work, however.

The trusted execution context must consider anything that it receives as untrusted. In particular, this holds when $T$ accesses the stable storage through load and store, in order to persist its state $M$. With a correct $S$, load always returns the state that has been stored most recently. For protecting against a malicious $S$, the trusted execution context uses encryption and authentication to protect $M$ before it leaves $T$. Yet, a malicious server may still return a correctly protected but outdated state to $T$. We call such a consistency violation a rollback attack. In particular, a malicious server may restart $T$ at any time and load its memory from some state that $T$ has stored earlier.

Furthermore, a malicious server may start multiple instances of a trusted execution context and let the clients interact with different instances over time. In this way, clients may be separated so that they only see operations of other clients talking to the same instance. Even if the TEE can run only a single $T$ at a time, $S$ can multiplex different copies of the trusted context. The malicious server might supply a different, but valid state to each trusted execution context instance, similar to a rollback attack. This clearly violates the consistency of the data, so that the responses from different trusted execution contexts to the clients diverge. We call this a forking attack; it is more general than a rollback attack because multiple instances of $T$ answer concurrently to the clients. Note that with a single instance of $T$ a forking attack always involves at least one rollback attack. It is well-known that clients cannot detect rollback and forking attacks in asynchronous systems, unless they communicate directly with 
each other [30].

\section{Protecting against Forking Attacks}

\subsection{Trusted monotonic counters}

For defending an execution context $T$ against a forking attack, we need to assure state continuity, i.e., that the state of $T$ evolves continuously and is never rolled back. One might think that $T$ could simply maintain a cryptographic hash of $M$ inside the TEE whenever it stores $M$ and verify that upon a load operation. However, this does not work because the memory of $T$ and the TEE is volatile and disappears when the epoch ends.

To overcome this, $T$ will need non-volatile storage that survives reboots. Such defenses have been proposed in the form of an attested append-only memory (A2M) [15] or a trusted incrementor (TrInc) [26]. These works demonstrate that the functionality needed from the trusted non-volatile storage can be reduced to a trusted monotonic counter (TMC).

In more detail, suppose $T$ has access to a TMC that is located in the TEE, the TMC uses a nonvolatile storage location that survives power loss, and the TMC's state and its communication with $T$ are protected from $S$. Whenever $T$ stores $M$ at the untrusted server, it increments the counter and includes the counter value with the state. When $T$ is restored, e.g. after a reboot, it loads its state from $S$, extracts the counter value, reads the TMC, and compares it to the extracted counter. Since $T$ protects all stored data cryptographically with a key known only inside the TEE, the server cannot tamper with the counter attached to $M$. This allows $T$ to detect rollback attacks. However, this approach suffers from several disadvantages as we argue now.

First, it is not easy to tolerate concurrent crashes and maintain liveness [32] at the same time; that is, when $T$ has incremented the TMC but the server crashes before the counter value has been saved to the non-volatile trusted area, then $T$ might falsely accuse the correct server of performing a rollback attack. The reason is $T$ cannot differentiate between a rollback attack and a server crash. In order to tolerate crashes, one can resort to complex schemes that ensure state continuity, which increment the TMC, save it persistently, and write state to disk atomically; they either need hardware modifactions [36] or perform a variation of 2-phase commit [32, 38], but the latter only works for deterministic operations, which can be replayed by $T$ and always give the same output.

Second, TMC-based solutions often suffer from limited performance. Typically, TMCs are implemented using TPMs which are well known to be slow [32]. The reason is that in order to prevent a counter overflow, the TMC artificially increases the time to increment the counter to several milliseconds. Although a response time of a several milliseconds is acceptable for, say, digital right management (DRM), this has a negative impact on the throughput of a server application that processes requests at a high rate.

Finally, the main disadvantage of any TMC-based approach is the lack of location transparency. That is, the TMC is normally bound to one trusted execution environment within one server. However, in modern cloud-computing platforms, applications must be able to scale and run on different servers during their lifetime. This may already be caused by system maintenance. For the end-user this should be completely transparent, but a trusted execution context cannot be stopped on one server and restarted on a different server with the same TMC; this is exactly what trusted hardware should prevent. Therefore this would require a migration protocol that needs the help of a trusted party.

For these reasons, we do not consider any solution that requires extra hardware or restricts the application to be deterministic in this paper. Instead we exploit the guarantees available with standard TEEs. 


\subsection{Ensuring consistency at the clients}

In the model considered here the TEE does not prevent a malicious server from mounting rollback and forking attacks and from isolating the clients from each other. The best possible option is to ensure that the clients remain "synchronized" with each other as much as possible and to mitigate attacks through this.

\subsubsection{Fork-linearizability}

Fork-linearizability [30] denotes the strongest consistency notion among the clients that can be achieved in the presence of rollback attacks and without client-to-client communication. This well-established notion ensures that whenever the malicious server has separated two clients, they can never be joined again to see mutually inconsistent responses from the server, without one of them detecting the attack. In essence, the server has to pretend that the inconsistency remains forever. Clearly, the clients can detect this though a lightweight, out-of-band mechanism.

Protocols that ensure fork-linearizability work by embedding information about the causal past of each operation into the requests from client to servers [30, 11, 7]. They use hash chains, Merkle trees, and vector clocks for representing the past history of operations and their context. Such protocols are very similar to the use of hash chains in blockchain platforms [6], cryptocurrencies such as Bitcoin, and Certificate Transparency [25].

The standard notion of linearizability [20] requires that the operations of all clients appear to execute atomically in one sequence, and that the atomic sequence respects the real-time partial order of the operations that the clients observe. Fork-linearizability is defined as an extension of this, which relaxes the condition of one sequence to permit multiple "forks" of an execution [30, 12]. Under forklinearizability, every client observes a linearizable history and when an operation is observed by multiple clients, the history of events occurring before the operation is the same. In this context, the view of a client $C_{i}$ denotes a correct, serialized history of operations for the functionality $F$, which includes all operations of $C_{i}$. For a more formal treatment we refer to the literature [12].

Unfortunately, fork-linearizability cannot be achieved without taking into account that some client operations on a correct server are blocked until other, concurrent operations terminate [12]. This inherent limitation has led to the relaxed notions, such as weak fork-linearizability. In FAUST [10], for instance, an operation returns a response to the client that is not guaranteed to be immediately fork-linearizable or linearizable, but the protocol notifies the client later when it knows that other clients have observed the operation as well. This is captured by the notion of stability, discussed next.

\subsubsection{Operation stability}

We now define a way to inform the client about those of its operations that have reached some level of consistency with respect to other clients. More precisely, we call an operation o by a client $C_{i}$ stable with respect to another client $C_{j}$ if the views of $C_{i}$ and $C_{j}$ both include $o$. In other words, $C_{i}$ knows that $C_{j}$ has observed $o$ and that $S$ was forced to take into account any effects of $o$ in later service responses to $C_{j}$.

Operation stability has also been used by [39, 10]. Here we use it as follows. We augment the response event of every operation with two numbers: a sequence number, which is assigned by the protocol to the operation that completes; and a stable sequence number, which denotes the latest stable sequence number of this client. The sequence numbers returned at one client are strictly increasing; the stable sequence numbers never decrease. 
Definition 1 (Operation stability). Let $o$ be a complete operation of $C_{i}$ that returns sequence number $t$. We say that $o$ is stable w.r.t. a client $C_{j} \neq C_{i}$ after $C_{j}$ completes any operation that returns a sequence number that is bigger than $t$. Operation $o$ of $C_{i}$ is always stable w.r.t. $C_{i}$.

For a set of clients $G$ that includes $C_{i}$, an operation $o$ of $C_{i}$ is stable w.r.t. the set of clients $G$, when $o$ is stable w.r.t. all $C_{j} \in G$. An operation that is stable w.r.t. all clients is simply called stable.

One may use different strengths of stability; for example, an operation might take a long time until it becomes stable (because all clients must observe it), but it might already be stable at a subset of the clients much earlier. A particularly useful subset is a majority quorum of the clients.

Definition 2 (Operation stability among a majority [35]). An operation $o$ of $C_{i}$ is stable among a majority of clients, when $o$ is stable w.r.t. a set of clients $C$, where $|C|>n / 2$.

Note that any subsequence of a history that contains only operations that are stable among a majority is linearizable.

\section{Lightweight Collective Memory}

This section introduces Lightweight Collective Memory (LCM), a protocol that allows a group of mutually trusting clients to run a service on a (potentially malicious) remote server. It benefits from a trusted execution context $T$ that runs on the server and executes the operations on behalf of the clients. LCM facilitates the detection of forking and rollback attacks against $T$ by ensuring fork-linearizability for every client operation. Moreover, LCM indicates which operations are stable among a majority; this permits clients to infer when their operations are linearizable. The LCM protocol benefits from the security guarantees of the TEE; in contrast to all previous protocols in the line of work originating with Mazières and Shasha [30], aiming at fork-linearizable semantics, the clients do not verify operation results here. Clients only handle metadata and rely on the TEE for producing correct responses.

\subsection{Overview}

LCM executes a stateful functionality $F$ inside a trusted execution context $T$ that is instantiated with the LCM protocol and also runs $F$. The trusted $T$ constructs a hash chain from the history of all operations that it executes and embeds this information in its responses to the clients.

A client invokes an operation by sending an encrypted INVOKE message to the (untrusted) server $S$, which forwards all incoming messages to $T$. After $T$ has decrypted this, it first verifies that the view of the client is consistent with $T$ 's own history. Then $T$ executes the operation and assigns a sequence number to it. The operation produces an output for the client and may modify the state of $F$. The output is returned to the client in a REPLY message, together with the sequence number and the latest stable operation (represented as a sequence number). When the client receives the REPLY message, it completes the operation and returns the result, the assigned sequence number, and the majority-stable sequence number. The latter informs the client about the stability of its earlier operations.

Fig. 2 shows the protocol interaction. For simplicity we assume that each client invokes operations sequentially, that is, it invokes a new operation only after completing the previous operation. For protecting $T$ against a malicious $S$, three cryptographic keys are used:

1. To safeguard the protocol's consistency data in the hash chain and the service state, $T$ encrypts it with a protocol-state encryption key $k_{P}$ before storing it in the server's stable storage and decrypts it again after a load. This key is generated by an admin during bootstrapping and required for migrating $T$ to another server. 


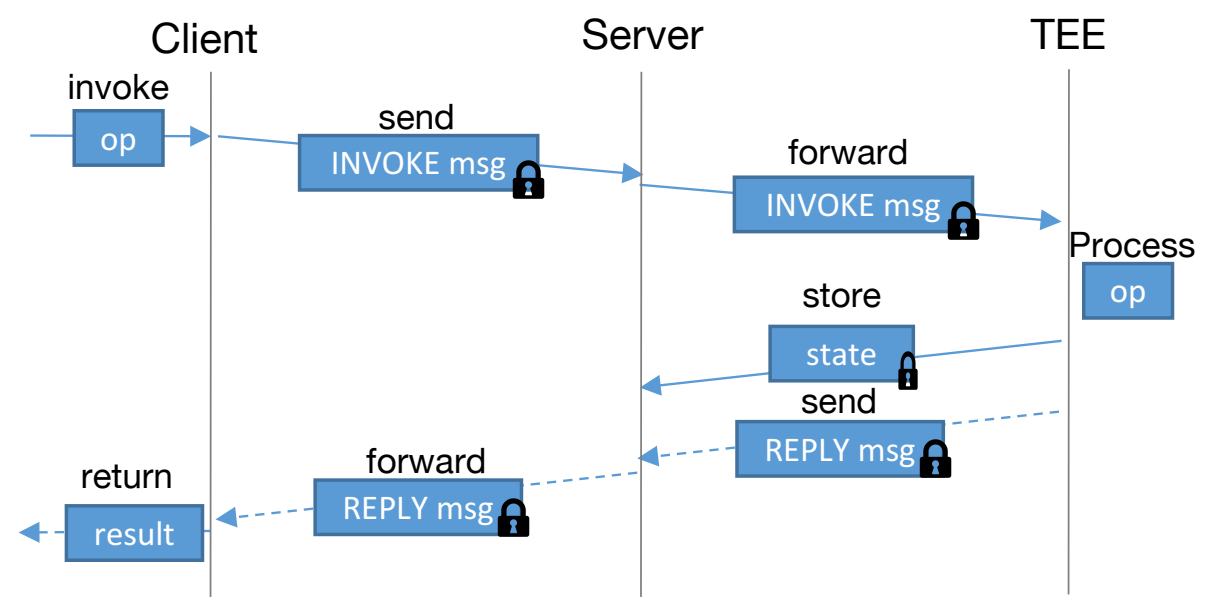

Figure 2. Protocol messages in Lightweight Collective Memory

2. A sealing key $k_{S}$ is initially generated by the TEE using get-key $y_{T, L C M}$ when $T$ starts. It encrypts the protocol-state key $k_{P}$ when $T$ stores this in persistent storage to tolerate crashes.

3. A communication key $k_{\mathcal{C}}$ protects all messages exchanged between the clients and $T$. The key is also generated by an admin and made known to all clients and to $T$.

All encryption operations use authenticated encryption with a symmetric-key $k$ and two functions auth-encrypt $(m, k)$ and auth-decrypt $(c, k)$ for a message $m$ and ciphertext $c$. Authenticated encryption produces a ciphertext integrated with a message-authentication code (MAC); it protects the content from leaking information to $S$ and prevents that $S$ tampers with messages or stored data by altering ciphertext. The hash function in LCM, denoted hash(), can be any cryptographically secure collisionfree hash function; it maps a bit string $x$ of arbitrary length to a short, unique hash value $h$.

\subsection{Protocol}

\subsubsection{Invocation at the client (Alg. 1)}

The client uses variables $t_{c}$ and $t_{s}$ to hold sequence numbers for the last operation by $C_{i}$ and the last operation stable among a majority, respectively. In addition, the client stores $h_{c}$, the hash chain value computed by $T$ corresponding to its most recent operation (with sequence number $t_{c}$ ). When $C_{i}$ invokes an operation $o$, it buffers $o$ in a variable $u$ and sends an encrypted INVOKE message containing $i, o, t_{c}$, and $h_{c}$. The latter two values represent the context in which $C_{i}$ invokes $o$; they result from $C_{i}$ 's last operation.

\subsubsection{Execution at $T(\mathbf{A l g} .2)$}

The trusted execution context $T$ maintains the sequence number of the most recently executed operation in a counter $t$ and a corresponding hash-chain value in $h$. T processes the operations of the clients sequentially. When $T$ receives an INVOKE message from $C_{i}$, it decrypts the message with $k_{\mathcal{C}}$ and signals a violation if the message does not have valid authentication. Then $T$ verifies that $\left(t_{c}, h_{c}\right)$ sent by the client correspond to the last operation response that $T$ has returned to $C_{i}$. For this purpose, $T$ maintains a map $V$ indexed by client identifier, where entry $V[i]$ holds the sequence number of the last acknowledged operation by $C_{i}$, the sequence number and corresponding hash chain value after the last operation by $C_{i}$. Again, when an inconsistency is detected, then $T$ halts. This verification is essential for 
the protocol and has three goals: First, it acknowledges the previous operation by $C_{i}$ in the sense that $T$ learns that $C_{i}$ has actually received the reply for its last invocation. Second, this detects message-replay attacks. When a malicious $S$ forwards the same INVOKE message multiple times, $T$ can easily filter these out with $V$. Finally, the verification detects rollback or forking attacks because the client sends the condensed view of its own history contained in $t_{c}$ and $h_{c}$.

If sequence number and hash chain value verification is successful, then $T$ increments the sequence number $t$, and calls exec $F$, which applies the operation $o$ to state $s$ and yields the corresponding result $r$ according to $F$. Next, $T$ extends the hash chain $h$ by setting this to hash $(h\|o\| t \| i)$. With the information from the INVOKE message, $T$ also determines if more operations have become stable. It uses the data in $V$ and a function majority-stable that returns $q$, the highest sequence number of an operation stable among a majority.

Then $T$ sends a REPLY message to $C_{i}$ encrypted with $k_{\mathcal{C}}$, containing the sequence number $t$, the hash chain value $h$, the result $r$, the stable operation $q$, and the client's previous hash chain value $h_{c}$. Before sending REPLY, $T$ also needs to store the current state for recovering from a crash. For this, $T$ encrypts the service state $s$, the protocol state $V$, and the key $k_{\mathcal{C}}$ using auth-encrypt with $k_{P}$ and stores this as a blob through $S$.

\subsubsection{Verification at the client}

When $C_{i}$ receives a REPLY message, it uses $k_{\mathcal{C}}$ to decrypt the contents and extracts $t, h, r, q$, and $h_{c}^{\prime}$. The client verifies that the previous hash chain value $h_{c}^{\prime}$ is equal to its own $h_{c}$, in order to match the REPLY message to its most recent INVOKE. Next, $C_{i}$ stores the new sequence number and hash chain value $(t, h)$ and outputs the operation result $r$ and the majority-stable operation $q$. These two sequence numbers allow the client to keep track of the operation history. In particular, a majority of clients have observed all operations with sequence numbers up to $q$. Any operation of $C_{i}$ with the sequence number $t^{\prime} \leq q$ is now stable among a majority. For correct functioning of the protocol, the state of each client must be recoverable from stable storage if a client crashes. For simplicity this is not part of the pseudocode.

\subsubsection{Server}

The (correct) server $S$ runs a TEE and hosts $T$, which is initially created by an admin. Whenever $S$ reboots or crashes, it restarts $T$. Recall that a malicious $S$ may restart the trusted execution context at any time or even spawn multiple instances. Furthermore, a correct $S$ forwards all messages between the clients and the trusted execution context in FIFO order. A malicious server, in contrast, can discard, reorder or delay messages.

\subsubsection{Protocol details}

In the pseudocode in Alg. 1 2, the symbol $\|$ denotes the concatenation of bit strings, and the assert statement, parameterized by a condition (where $*$ matches any value), immediately terminates the protocol when the condition is false. The clients and $T$ use this to signal that the server misbehaved. Note that auth-decrypt may also signal an error; this is equivalent to an assert FALSE statement.

\subsection{Bootstrapping}

Bootstrapping sets up the necessary cryptographic keys and security contexts for trusted execution. It consists of three phases: (1) creating a trusted execution context $T$ on a remote server; (2) remote attestation and provisioning of $T$; and (3) key distribution among the group of clients. 


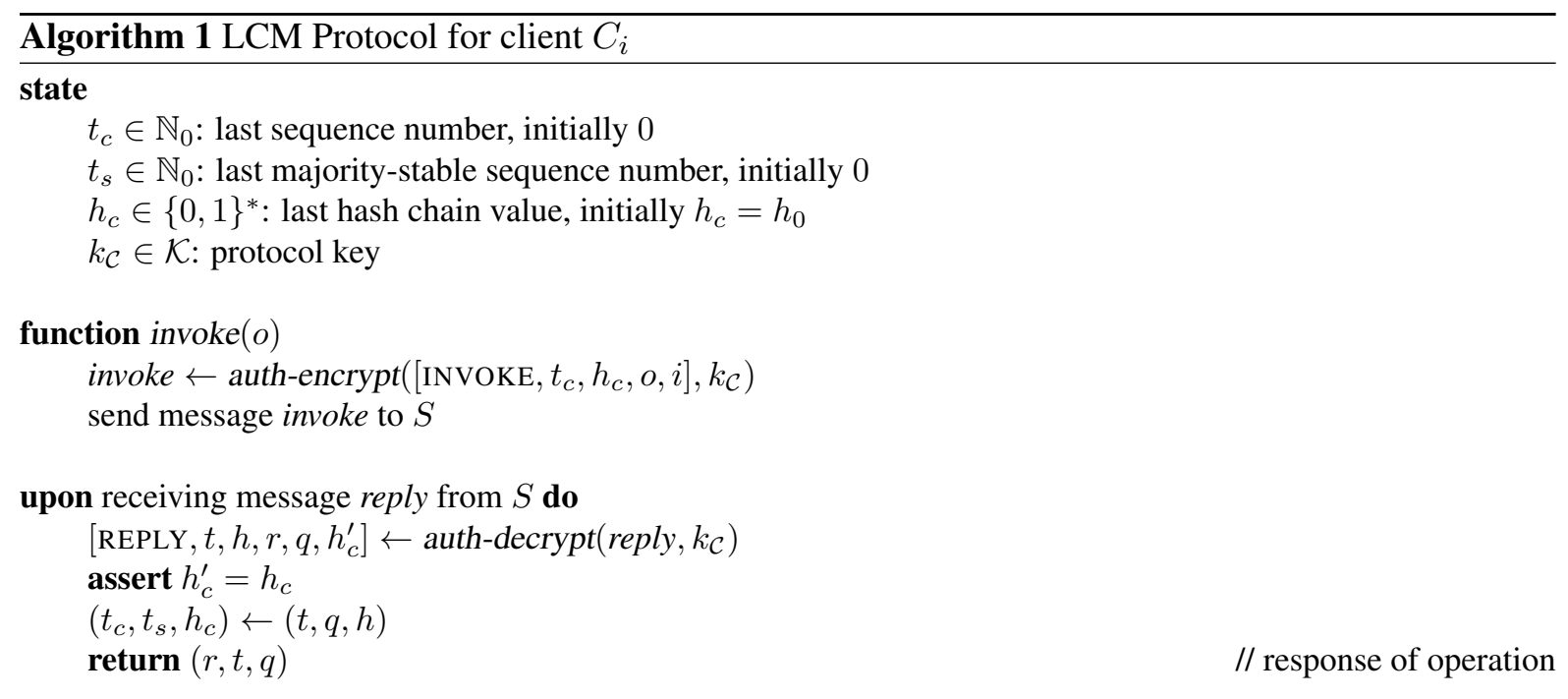

In the first phase, a special admin client instructs the server to create a new trusted execution context $T$ for running protocol LCM (Alg. 2). When $T$ starts this protocol, it enters init first. Function init is also executed after a reboot, where it first loads the encrypted state from stable storage. During initialization no such state exists yet.

Second, the admin initiates the remote attestation process, to verify that $T$ has been started correctly and is running LCM. Remote attestation is a core function of the TEE and produces a cryptographic proof, which convinces the admin that $T$ indeed runs LCM. If a malicious $S$ would instantiate $T$ with some $P \neq L C M$ this verification will reveal it. Note that the remote attestation protocol also convinces the admin that $T$ is actually executed on the TEE and protected against a malicious server.

Finally, the admin generates two secret keys, $k_{\mathcal{C}}$ for securing the communication and $k_{P}$ for storing the protocol state, and injects them into $T$ through a secure channel provided by the TEE. After $T$ has received the keys, it initializes the protocol and service states, and retrieves a sealing key $k_{S}=$ get-key ${ }_{T, L C M}$ from the TEE. Recall that $k_{P}$ is used to encrypt the state, and that $k_{P}$ and $k_{\mathcal{C}}$ together are stored encrypted $k_{S}$. Since $k_{S}$ is generated in a deterministic way in the trusted hardware of the TEE, $T$ can recover its state from an earlier epoch using the stable storage of $S$ after a crash. And because every $T$ running on a different physical TEE obtains a different sealing key, this binds the state of $T$ to the hardware. The admin also distributes the communication key to the clients using a secure channel to each of them.

\subsection{System reboot and recovery}

The server $S$ controls starting and stopping $T$. As argued before, the TEE is stateless and, therefore, $T$ cannot distinguish a reboot after a crash from an attack by $S$. In order to tolerate server crashes and reboots without administrative intervention, but also to facilitate planned restarts, the application state is stored on stable storage.

When the server reboots after a crash, it recreates the trusted execution context $T$ that runs LCM. $T$ then enters init, which first tries to load a previous state and resumes from there when it exists. As $T$ obtains $k_{S}=$ get-key $_{T, L C M}$ from the TEE, it can decrypt and authenticate $k_{P}$ and the state with $k_{S}$; the state also contains $k_{S}$ for communicating with the clients. $T$ recovers $V$ form the state and can easily derive $(t, h)$ from $V$ by looking up the client which executed the last operation in $V$. Formally, $V$ is an array of $\left(t_{a}, t, h\right)$ triples, and $\operatorname{argmax}(V)$ returns the index of the triples with the highest sequence 


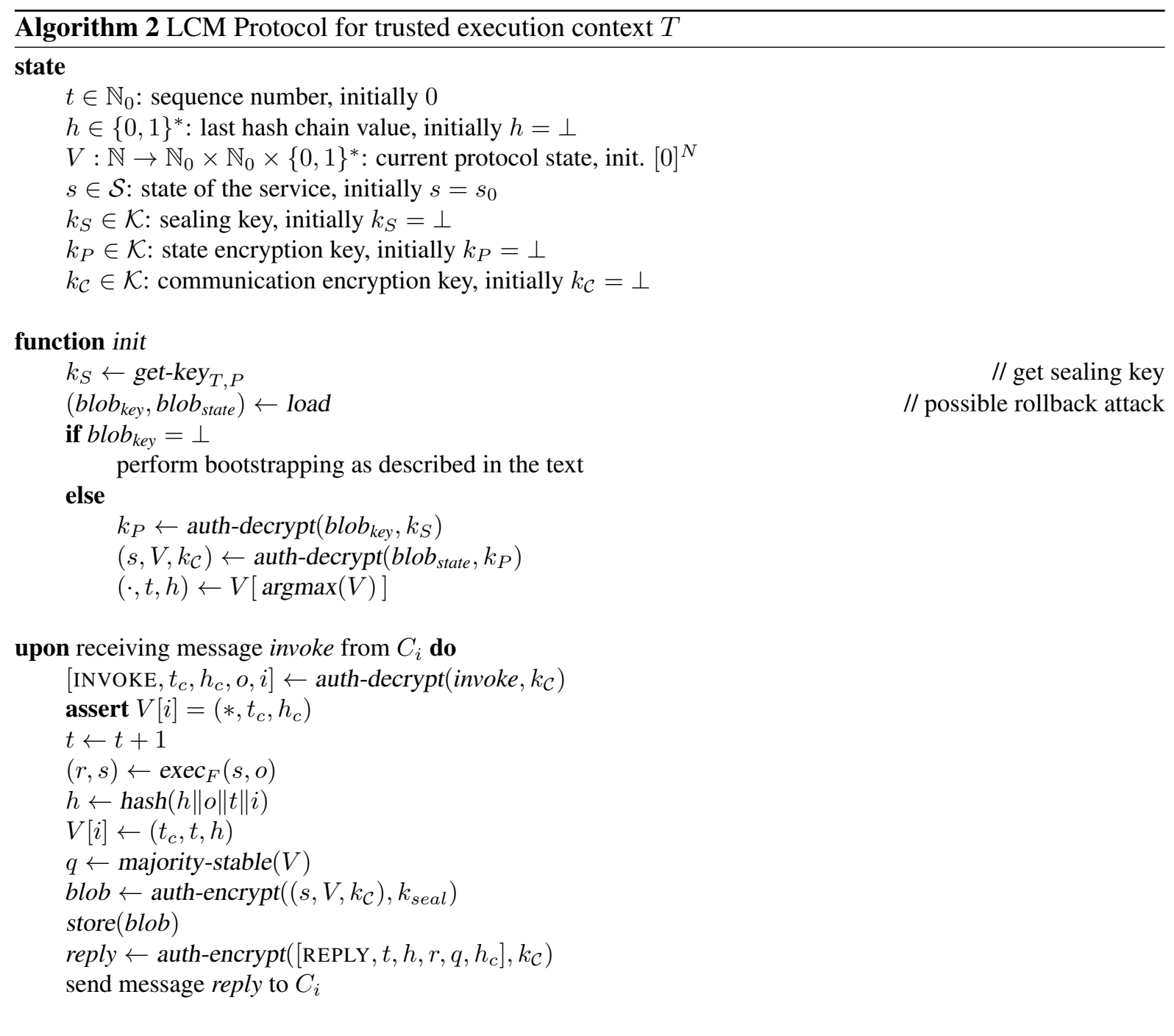

number $t$.

$T$ has now entered a new epoch and is ready to continue request processing without remote attestation. The clients trust that $T$ runs LCM from the initial verification step during bootstrapping and from the binding of the sealing key $\left(k_{S}\right)$ to the TEE through the secure hardware. Recall that $T$ recovers the communication key $k_{\mathcal{C}}$ via the sealing key. Once a client can engage in encrypted and properly authenticated communication, protected through $k_{\mathcal{C}}$, to some TEE, the trust of the client from the initial attestation extends to the current holder of $k_{\mathcal{C}}$.

\subsection{Stability}

For determining the stability of operations, $T$ maintains the map $V$ with two sequence numbers for every client. One sequence number of the last acknowledged operation, and another sequence number of the last operation. The function majority-stable $(V)$ returns the sequence number of the operation that is stable among a majority, that is, the largest acknowledged sequence number in $V$ that is less than or equal to more than $n / 2$ sequence numbers in $V$. Stability indicates to the clients when their operations have been observed by others and helps detecting forking attacks. When the server is correct and all clients periodically invoke operations, then all operations become stable eventually. In the case of a 
forking attack, where one or more clients are separated, the operations of the forked clients will cease to become stable.

The client protocol returns the sequence number $t$ and the majority-stable sequence number $q$ together with the operation result. This enables the client to track the progress of the operation history. Depending on the application, a client might want to verify that some critical operation has become stable or wait until it does before invoking new operations. Note that the client protocol as described in Alg. 1 1 only receives stability updates when it invokes new operations. If the client needs to be informed earlier about the stability of past operations, it can simply invoke dummy operations periodically, as introduced by FAUST [10]. Alternatively, Alg. 1 1 could be extended to support a callback mechanism, where clients can register for notifications of stability updates, as also used in Venus [35].

\subsection{Extensions}

\subsubsection{Tolerating server crashes}

As the server might crash, we now extend the protocol to allow $T$ to recover and continue processing. In the simple case where $T$ crashes while it is idling, the correct server restarts it and continues with the protocol as described before. On the other hand, when $T$ crashes during the processing of a client request, we differentiate between two cases: either it crashes before the store operation returns and has saved the application and protocol state or afterwards.

Therefore, we equip the client with a retry mechanism: When the client has not received a reply until a timer expires, it sends the message again, but marks it as a retry attempt. In the first case ( $T$ crashes before successfully stores), the server will restart $T$ and it eventually receives the retry message. The verification of the sequence number $t_{c}$ and the hash chain value $h_{c}$ ensures that the lost message has not already been processed. $T$ simply continues processing and returns the reply to the client. In the second case (when $T$ crashes after stores), the verification of $t_{c}$ and $h_{c}$ fails since $t_{i}$ stored in $V[i]$ is bigger than the value received from $C_{i}$. The retry marker instructs $T$ to not consider this as a rollback attack. Therefore, we extend the protocol state $V$ to store the last operation result $r$ as well. Then $T$ can retrieve the result from $V$ and (re)send the REPLY message.

\subsubsection{Server migration}

Since location transparency is a major advantage in cloud computing, we also include a migration mechanisms that allows to move a trusted execution context $T$ to a different host system. There are two trusted execution context instances involved, $T$ on the origin system and $T^{\prime}$ on the target system to which the protocol migrates. Migration requires cooperation between the two machines and that the server's stable storage can be accessed from the origin and the target system, for instance by using shared remote storage.

The migration works as follows. The (correct) origin server signals the target server to start a trusted execution context $T^{\prime}$ and to prepare it for migration. Normally, $T^{\prime}$ would try to retrieve a state encryption key $k_{P}$ from stable storage but since it was encrypted with the sealing key of $T$ on the origin system, $T^{\prime}$ cannot obtain it. For this reason, $T$ takes over the role of the admin and bootstraps $T^{\prime}$ according to the earlier description. After a successfully remote attestation, $T$ injects the state encryption key $k_{P}$ via a secure channel. At this point, $T$ stops processing requests and provides its current state to $T^{\prime}$; then $T^{\prime}$ restores the application and protocol state, resumes executing requests, and is still able to uphold the guarantees of LCM against rollback and forking attacks.

This migration mechanism does not require a trusted party and works completely transparently for the clients. However, when the origin system crashes without any possibility to recover, e.g., when the TEE hardware malfunctions, then an intervention by a trusted admin is required. In contrast to the 
solutions based on a TMC mentioned in Sec. 3.1, this migration mechanism is more robust to server failures. In particular, the migration of a TMC always requires an admin to read the last TMC value from the origin system and to update the TMC on the target system with the correct counter value. Clearly, this fails if the origin system becomes inaccessible. LCM still allows migration because the TEE is stateless and because the state is stored on remote storage. Our proposed migration scheme is similar to [37].

\subsubsection{Group membership}

In a practical system, the group of clients will dynamically change, as clients may be removed from the collaboration group and new clients may join. Although the protocol formulation uses a static client group, it is easy to extend LCM for handling dynamic changes. When a new client joins the group, the admin sends the shared secret $k_{C}$ for secure communication with the trusted execution context to the new client and instructs $T$ to include the client in the protocol state. For removing a client, the admin generates a new fresh communication key $k_{C}^{\prime}$ and distributes it to all remaining clients. Then the admin sends a removal request with $k_{C}^{\prime}$ to $T$, which uses the fresh key afterwards.

\section{Implementation}

The LCM protocol relies on our assumptions as described in Sec. 2 and can be implemented with any TEE technolgy such as Intel SGX.

\subsection{Intel SGX}

Intel's Software Guard Extensions (SGX) [31] adds hardware enforced security to the Intel CPU architecture. SGX enables applications to execute certain code in a trusted execution context, also called enclave. Enclaves are isolated and a hardware enforced mechanism guarantees the confidentiality and the integrity of an enclave even if the entire system is compromised. Moreover, the SGX platform checks that an application has not been tampered with when loading code and data at initialization into an enclave. SGX offers an attestation mechanism [1] for enclaves that allows to prove to a remote third party that an enclave runs a given application on an actual SGX platform. For utilizing the system's persistent storage and at the same time preserving data confidentiality and integrity, SGX supports data sealing. It permits to unseal data only by the origin enclave or another enclave by the same enclave developer. In the SGX programming model, applications in an enclave are considered to be trusted whereas all other applications (even the operating system) are untrusted. Typically, those enclave applications are small, hence, it is less likely to expose vulnerabilities. Using the SGX Software Development Kit (SDK) [23, 22] enables developers to divide their applications into a trusted component (enclave) and untrusted component. The trusted component is signed by the developer. For bridging the trust border between enclaves and untrusted components, SGX provides the Enclave Definition Language (EDL) that is used by enclave developers to specify an interface and generate "gateway" code comprising Enclave calls (ecall) and Outside calls (ocall).

\subsubsection{Enclave protection}

SGX features two properties that are essential to execute code securely in an enclave. First, SGX verifies that an enclave is instantiated with the correct application. The enclave code contains an Enclave Signature (SIGSTRUCT) produced by the enclave developer that allows the SGX platform to detect whether 
the code of the enclave has been tampered with. In particular, SIGSTRUCT comprises an enclave measurement (a cryptographic hash that identifies the code and data), a signature over the measurement, and the enclave developer's public key, that serves as the identity of the enclave developer. When the enclave is loaded, the CPU verifies the signature and calculates the enclave measurement and compares it to the measurement in SIGSTRUCT; if they match the enclave completes its instantiation successfully. Second, SGX protects against any access and modification from untrusted components. To this end, the enclave resides in an isolated memory area called enclave page cache (EPC) that can not be accessed from outside an enclave. This is enforced through a memory access control mechanism. The EPC size is limited to $128 \mathrm{MB}$, thus, when enclave reaches that limit or a context switch occurs, pages are moved to DRAM. A memory encryption engine [19] protects pages when swapping between EPC and DRAM in terms of confidentially, integrity, as wells provides replay attack prevention. Those two mechanisms prevent any untrusted component from accessing or modifying the enclave memory. Note that this mechanism only protects the in-memory state but not persistent state of an enclave. When an enclave is terminated, all in-memory state is lost.

\subsubsection{Enclave attestation}

SGX supports remote attestation [1] that demonstrates to a remote client that an enclave runs a given application inside a SGX platform and therefore can be considered to be trustworthy. This is vital for establishing trust in an enclave application and is required prior provisioning any secrets or protected data. The remote attestation briefly works as follows: A remote client sends a challenge to the enclave including a nonce. The enclave produces a report that comprises some metadata including a short hash value of the application code, the enclave developer identity, and additional user data. The user data contains the nonce. Note that enclave developers may also include custom values in the user data, for instance, some information about the current enclave state. Additionally, the report comprises a MAC that is produced using a report key provided by the SGX platform. A special enclave, so called Quoting enclave, receives the report and validates it by using the same report key. The SGX platform enforces that only enclaves are able to retrieve this report key, thus, are able to create and verify report structures. If the verification succeeds, the Quoting enclave signs the report with a platform specific key and replaces the MAC with the signature. SGX leverages a group signature scheme (EPID [9]) that does not reveal the identity of the platform. In other words, the signature states that some SGX platform has produces that signature. The signed report (Quote) is sent to the remote client which then validates signature (using an EPID infrastructure), verifies integrity of the attest, and finally checks that that the Quote matches the challenge using the nonce.

\subsubsection{Data sealing}

Application code and data are secured while residing within an enclave. However, when an enclave is terminated the data is lost and can not be recovered when the enclave restarts again. Therefore, SGX features a sealing mechanism [23, 22] based on AES-GCM-128 that allows to encrypt and authenticate data before it leaves an enclave by using a special sealing key provided by the SGX platform. In particular, SGX provides two types of sealing: An enclave identity based sealing that only allows enclaves running the same application to unseal the data; and enclave developer based sealing where all enclaves, which are developed (signed) by the same developer, can unseal the data.

\subsection{LCM framework}

We implemented LCM as a framework in Java and C++ consisting of a client-side and a server-side library that can be integrated with SGX-enabled applications which require rollback and forking detection 
for persistent state. The LCM client-library is implemented in Java and follows the description as presented in Alg. 1. It uses AES-GCM with 128-bit keys provided by the Java Cryptography Extension to protect the confidentiality and integrity of all protocol messages. The LCM client-library uses a simple network interface including methods for sending and receiving protocol message. This allows to reuse an existing application network stack instead of handling the communication with the server by our library. The LCM server-side library is implemented in C++ using the Intel SGX SDK (Version 1.6) [23]. It only utilizes trusted libraries provided by the SGX SDK, such as libsgx_tcrypto for cryptographic hashing and encryption. In particular, we use SHA-256 for constructing the hash chain and AES-GCM with 128-bit keys for encrypting the protocol messages, as well as the protocol and application state. The state encryption key is encrypted using the SGX sealing function before storing persistently. We defined two interfaces that must be implemented by the enclave application. First, an operation processor, that receives a client operation and returns the operation result; and second, a serialization interface that returns the application state as a byte sequence. The implementation does not strictly follow the Alg. 2 as presented in Sec. 4. That is, we optimized the code in order to eliminate the ocall when storing the application and protocol state at server's persistent storage. Instead, we piggyback the encrypted data together with the reply message. Furthermore, we implemented operation batching mechanism where the LCM protocol receives multiple invoke messages with a single ecall. In contrast to Alg. 2, the application and protocol state is stored once per batch. Our current proof of concept does not make use of remote attestation. However, this can be easily extended using the mechanisms as provided by the SGX SDK.

\subsection{Building applications with LCM}

In order to demonstrate our LCM framework we integrated LCM with a simple persistent key-value store (KVS) running in an enclave on a remote server. The prototype architecture is shown in Fig. 3 . Clients and the server communicating via TCP socket connections. A KVS stores data objects in a flat namespace, where each object is identified by a unique name or key. The KVS is implemented using trusted libraries provided by the SGX SDK. In particular, we use std::map for storing key-values pairs as strings of arbitrary length. The current version of the SGX SDK does not support std::unordered_map which would be our first choice due to its constant access time.

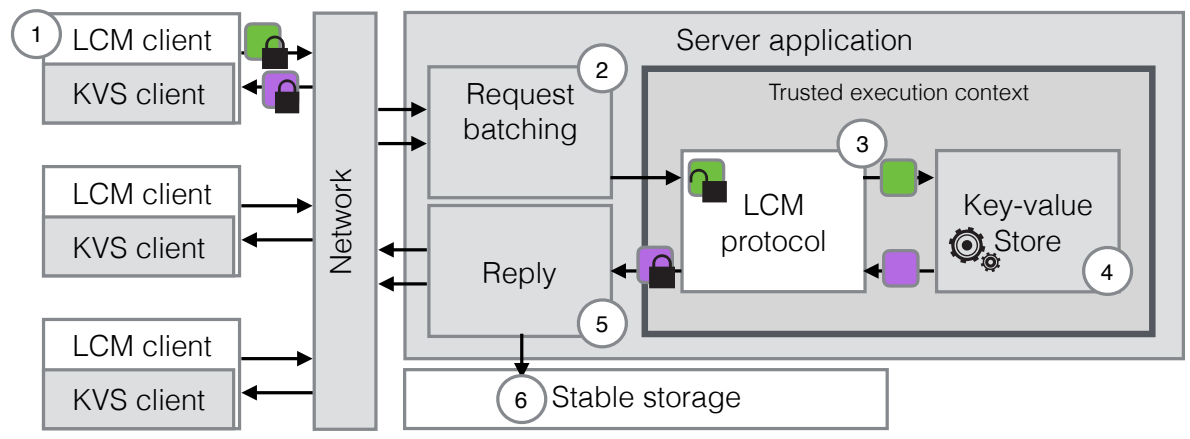

Figure 3. The prototype architecture of an enclave based key-value store protected with LCM.

Clients invoke GET, PUT and DEL operations through the KVS client which instantiates the LCM client-library. A server application handles the socket communications, implements an interface for stable storage (disk), and hosts an enclave running the server-side LCM protocol and the KVS. When the server application receives a client request (INVOKE message), it collects the message in a bounded queue (batch). Once the queue has reached the limit or no more client request are available, the server 
application performs an ecall and passes the collected (batched) messages to the enclave. The LCM protocol processes them sequentially and returns the corresponding REPLY messages for each client request and the aggregated application and protocol state. The server application then, writes the encrypted states to disk and forwards the REPLY messages to the clients. Note in order to achieve crash tolerance, the server application has to write the state synchronously to disk (fsync), this clearly decreases the performance. Our prototype implementation of a LCM-protected key-value store comprises about 4000 sloc, where enclave components comprise around 2200 sloc. The rest is for the untrusted server implementation including the storage and network code. The KVS client and the LCM client-library add additional 1600 sloc to the prototype.

\section{Evaluation}

We evaluated the overhead of LCM with a set of benchmarks using YCSB and compare it against a SGX-secured key-value store without rollback and forking protection. Furthermore, we compare the performance of LCM against a trusted monotonic counter approach and unprotected Redis.

\subsection{Experiment setup}

The experiments use a Dell Optiplex 7040 desktop machine with an i7-6700 Intel CPU that is SGXcapable to run the server. It is equipped with $8 \mathrm{~GB}$ of memory, 1 Gbps network connection and a SSD drive. We simulate clients on a virtual machine with 24 virtual CPUs and 8 GB of memory, running YCSB as workload generator using Oracle Java (JRE 8, build 1.8.0_111-b14). All machines run Ubuntu Linux 14.04.4 Server with the generic 4.4.0-47 Linux kernel. The evaluation is driven by $Y C S B$ [16], an extensible tool for benchmarking key-value stores. It supports many different key-value stores, such as Redis (http://redis.io), Cassandra (https:// cassandra.apache.org/) and many more. YCSB comes with a set of core workloads spanning different application scenarios. For the evaluation we use workload A with a mix of 50/50 PUT and GET operations and show the overall throughput of all clients. Every reported data point is taken over a period of 30 seconds. We integrated the KVS client including the LCM client-library with YCSB. As a baseline for our experiments we use our KVS (see Sec. 5.3) protected with SGX. For the comparison with Redis and our native KVS implementation we use Stunnel (https://www.stunnel.org) to secure the communication with the clients. Redis has been originally designed for deployment in private networks, thus, it does not support TLS connections. LCM and the SGX-based KVS prototype establish a secure communication with the clients by using AES-GCM encryption with 128 bit keys. In order to simplify the evaluation process we use predefined encryption keys.

\subsection{Enclave memory}

In a preliminary experiment we evaluated the memory consumption of the SGX key-value store. We inserted one million objects and measured the enclave heap allocation using $s g x$-gdb. Each object with a key size of 40 byte and 100 byte values. For 300000 objects we measured an allocation of $93 \mathrm{MB}$ enclave memory whereas we expected only about $40 \mathrm{MB}$. It turned out that the KVS implementation based on std::map;std::string, std::string; comes with a memory overhead of about $134 \%$. In particular, the string key-value pairs consume about 280 byte whereas the map data structure allocates additional 48 byte for each object for maintaining an internal search structure. Moreover, we measured the latency of PUT and GET operations for different number of objects. As the EPC is limited, we expected a performance drop when the number of objects increases and the SGX driver starts swapping EPC pages as also reported in [2, 8]. We observed that the latency increases up to $240 \%$ when the KVS holds 


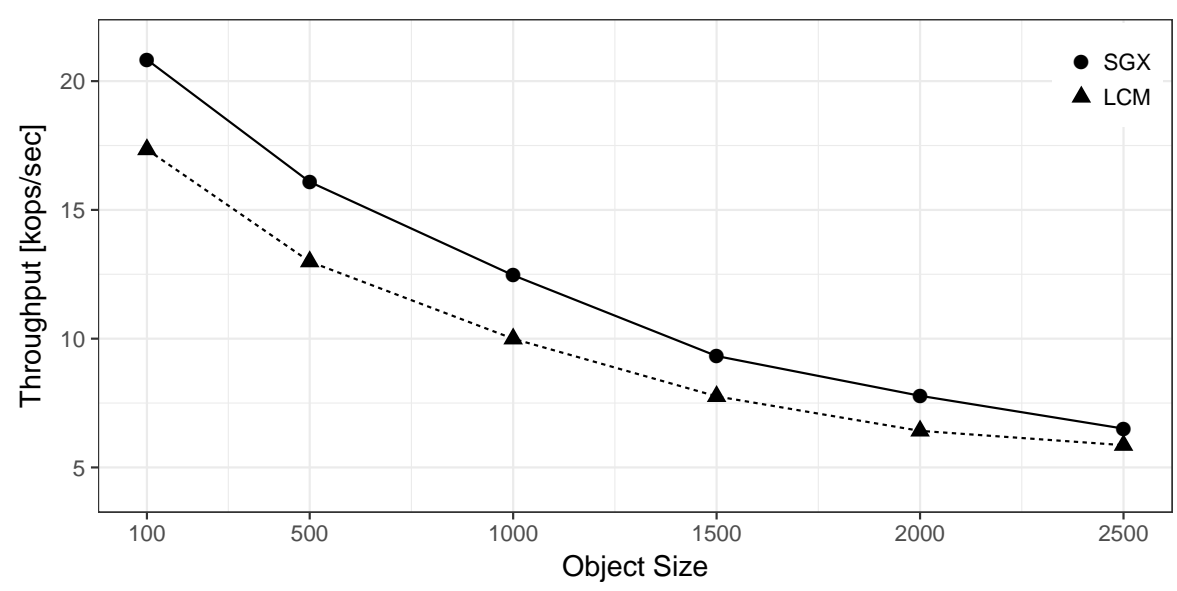

Figure 4. Throughput with different object sizes with async disk writes.

more than 300000 objects. We refrain from showing the graph due to page reasons. We assume that this hardware restriction will be addressed in future CPU releases and thus choose our further evaluation workloads to fit into the EPC.

\subsection{LCM protocol message}

We first study how the LCM protocol message overhead affects the throughput. As described in Sec. 4.2, LCM sends additional metadata, such as the sequence number and hash chain value, along with a client request. In particular, our LCM implementation adds 45 byte to an operation invocation and 46 byte to a result. This overhead remains constant for varying operation and result sizes. In order to evaluate this, we run the experiment with 8 clients for 1000 objects of size 100 to 2500 byte. Fig. 4 shows that the throughput of LCM behaves similar to the plain SGX KVS. As expected, we observe that LCM introduces an overhead but it decreases with bigger object sizes. In particular, for objects with the size of 100 byte the throughput is $20.12 \%$ and for objects with size of 2500 byte it is $10.96 \%$ lower compared to the plain SGX KVS.

\subsection{The throughput of LCM}

We also study the overall throughput of LCM by increasing the number of clients. This workload uses up to 32 clients, 1000 objects with a fixed size of 100 byte. Each object key is 40 byte. In this experiment we compare two variants of LCM against a KVS without SGX ("Native"), SGX-secured KVS ("SGX"), Redis, and SGX-secured KVS with emulated trusted monotonic counter ("SGX+TMC"). We run LCM and SGX without batching enabled and with batching of up to 16 operations. We configured Redis to use an append log strategy for persistence. We also disabled fsync (synchronous disk writes) for Redis as well as for our KVS prototypes. As Fig. 5 shows, the throughput of Redis and the Native KVS scale almost linear. In contrast, LCM and SGX reach saturation already with 8 clients. We observed that the SGX KVS reaches $0.42 \mathrm{x}-0.78 \mathrm{x}$ the throughput of the Native KVS. LCM, on the other hand, reaches $0.67 x-0.95 x$ the throughput of the SGX KVS, with batching even $0.72 x-0.98 x$. The reason is, LCM and SGX are single threaded applications and perform the encryption of every client request inside the enclave. Although, Redis and Native KVS are also single threaded, they leverage Stunnel that uses multiple processes to encrypt/decrypt client communication. That way, secure communication becomes a bottleneck. 


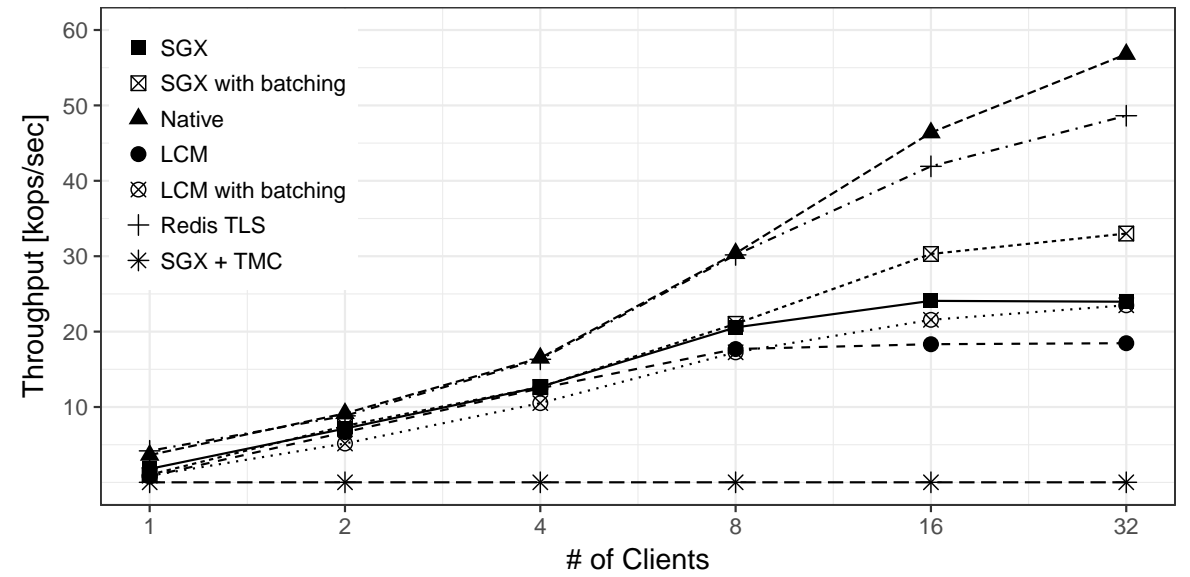

Figure 5. Throughput with different numbers of clients with async disk writes.

\subsection{The performance impact of Trusted Monotonic Counter}

In this experiment we investigate the performance impact of Trusted Monotonic Counters (TMC) when used to protect against rollback and forking attacks. The current version (Version 1.6) of the SGX driver and SDK do not yet support Intel's Trusted Monotonic Counter [23] on Linux. However, on Windows [22] they are available provided by the Intel management engine (ME) that stores the counter in non-volatile memory. We measured an average latency of $60 \mathrm{~ms}$ to increment a SGX TMC on Windows, whereas [38] reported even higher latency of about $95 \mathrm{~ms}$. We emulated the TMC on Linux by using a simple counter followed by setting the thread to sleep for $60 \mathrm{~ms}$ when incrementing the counter. As Fig. 5 shows, the throughput remains constant for the emulated TMC with an average of 12 operations per seconds, wheres LCM with batching, on the other hand is $96 x-2063 x$ faster. However, by using trusted monotonic counters rollback and forking attacks can be detected immediately but this comes with low throughput.

\subsection{The costs of crash tolerance}

Finally, we study the performance overhead induced by synchronous disk writes when storing the application and protocol state that is necessary to support crash tolerance. We performed the same experiment as in Sec. 6.4 but enabled fsync for our KVS prototypes as well as for Redis. We expect much lower performance compared to asynchronous writes. Fig. 6 shows the throughput with different number of clients with synchronous storing. As expected, fsync introduces high latency when writing to disk. In particular, we observed that throughput of Native, SGX, LCM, and SGX with TMC remain constant whereas Redis, SGX and LCM with batching scale. SGX KVS achieves 0.98x of the Native KVS throughput and LCM without batching achieves 0.69x of SGX KVS throughput. In contrast, LCM with batching reaches $0.72 x-9.87 x$ the throughput of the SGX KVS and $0.71 x-0.75 x$ the throughput of SGX KVS with batching. The experiment shows, that expensive storing operations reduce the relative overhead introduced by SGX but can be reduced by leveraging batching mechanisms.

\section{Related Work}

With the advent of SGX, trusted computing has achieved a new level of practicality with the aim of wide-spread deployment. Recent publications detail how legacy applications [4], micro services [2], 


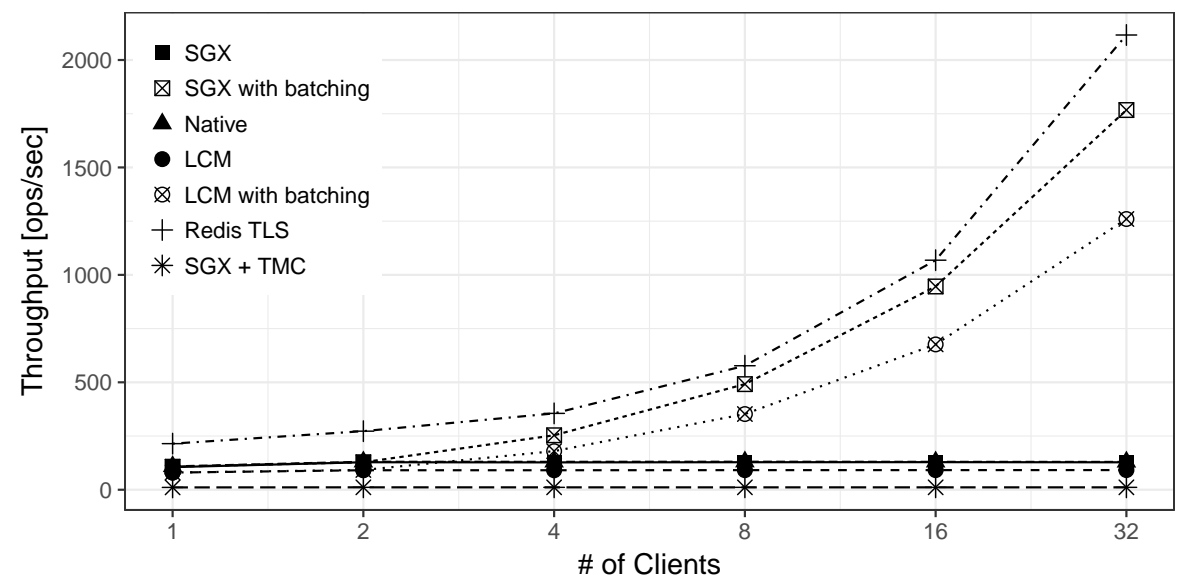

Figure 6. Throughput with different numbers of clients with sync disk writes.

data intensive programming [34] but also specific services [8] can be secured on top of an infrastructure where only the CPU needs to be trusted. While SGX provides special means to detect memory replay attacks against the enclave [19], external memory remains unprotected. Accordingly, additional measures are necessary to prevent rollback and forking attacks mounted through external memory and secondary storage. The latter is especially complicated if an enclave is restarted (e.g. due to crashes or system maintenance reboots). As a pragmatic solution, the Windows SGX SDK [22] offers trusted counters that are linked non-volatile memory inside the Intel management engine (ME). However, trusted non-volatile counters as provided by a TPM are slow, e.g. adding 35-95 ms latency for each operation depending on the hardware platform, as different reports show [38, 26, 29]. Thus, in essence, all hardware-based solutions that rely on trusted counters and are consulted on every request of a secured service suffer from performance problems [14, 26, 32]. An additional issue of current trusted counter TPM-based realizations is wear out if used very frequently. Strackx and Piessens [38] specifically address this problem by clever usage strategies, however the performance problems remain. Recent work [29] proposes a complementary approach to LCM where enclaves across multiple systems assist each other in order to prevent rollback attacks. This requires multiple enclaves to interact with each other to store and retrieve version information from the group of enclaves wheres in LCM it is stored at the clients.

Another line of work addresses the problem of rollback and forking attacks without relying on trusted components. With only a single client, the classic approach [5] for memory checking uses a hash tree where the client stores the root. Many systems build on this approach to protect remote storage services (e.g., Athos [18]). In the multi-client model, Mazières et al. [30] introduced the notion of fork-linearizability and implemented SUNDR [27], which confines rollback attacks to always present a view to each client that is consistent with its past operations; thereby fork-linearizability makes it much simpler to detect integrity and consistency violations on remote file storage. Cachin et al. [12] improved the efficiency of SUNDR and proved that there is no wait-free emulation of fork-linearizable storage. That is, sometimes clients are blocked until an operation by another client has finished. Systems such as SPORC [17], FAUST [10], and Venus [35] avoid blocking by weakening the consistency guarantees. Others have explored aborting operations [28, 11] and improved the efficiency by reducing the computation and communication overhead [7]. Mobius [13] uses forking properties in the context of disconnected operations. Previous systems have explored the guarantees of fork-linearizable for different applications [17, 40] and generic services [11].

LCM combines the best features of these two technologies, trusted execution environments and protocol-enforced consistency. It also addresses rollback and forking attacks on TEEs as much as pos- 
sible without introducing impractical limitations into a service.

\section{Conclusion}

This work has focused on a shortcoming of trusted computing technology, which affects current trusted execution environments (TEEs), such as Intel SGX. In particular, the trusted execution contexts or "enclaves" are stateless, lose their memory when a crash occurs, and need support from the host for state continuity. But since the host is also the adversary of the TEE according to the security model, it is actually impossible to implement protocols that survive crashes seamlessly and prevent rollback attacks at the same time without introducing extra hardware.

As a solution we have introduced Lightweight Collective Memory, a system for detecting rollback and forking attacks that ensures the consistency notion of fork-linearizable and determines when operations become stable. The LCM protocol complements TEE technology with a lightweight mechanism for maintaining consistency information by the clients.

\section{Acknowledgments}

We thank Anil Kurmus, Cecilia Boschini, Manu Drijvers, Kai Samelin, David Barrera and Raoul Strackx for interesting discussions and the anonymous reviewers of DSN 2017 for valuable comments. This work has been supported in part by the European Commission through the Horizon 2020 Framework Programme (H2020-ICT-2014-1) under grant agreements number 644371 WITDOM and 644579 ESCUDOCLOUD and in part by the Swiss State Secretariat for Education, Research and Innovation (SERI) under contracts number 15.0098 and 15.0087.

\section{References}

[1] I. Anati, S. Gueron, S. Johnson, and V. Scarlata. Innovative technology for CPU based attestation and sealing. In Int. Workshop on Hardware and Architectural Support for Security and Privacy (HASP), 2013.

[2] S. Arnautov, B. Trach, F. Gregor, T. Knauth, A. Martin, C. Priebe, J. Lind, D. Muthukumaran, D. O'Keeffe, M. L. Stillwell, D. Goltzsche, D. Eyers, R. Kapitza, P. Pietzuch, and C. Fetzer. SCONE: Secure Linux containers with Intel SGX. In USENIX Proc. on Operating Systems Design and Implementation (OSDI). USENIX Association, 2016.

[3] H. Attiya and J. Welch. Distributed Computing: Fundamentals, Simulations and Advanced Topics. Wiley, second edition, 2004.

[4] A. Baumann, M. Peinado, and G. Hunt. Shielding applications from an untrusted cloud with Haven. In USENIX Proc. on Operating Systems Design and Implementation (OSDI). USENIX Association, 2014.

[5] M. Blum, W. Evans, P. Gemmell, S. Kannan, and M. Naor. Checking the correctness of memories. Algorithmica, 1994.

[6] J. Bonneau, A. Miller, J. Clark, A. Narayanan, J. A. Kroll, and E. W. Felten. SoK: Research perspectives and challenges for Bitcoin and cryptocurrencies. In IEEE Proc. on Security \& Privacy, 2015. 
[7] M. Brandenburger, C. Cachin, and N. Knežević. Don't trust the cloud, verify: Integrity and consistency for cloud object stores. In Int. Systems and Storage Conference (SYSTOR). ACM, 2015.

[8] S. Brenner, C. Wulf, M. Lorenz, N. Weichbrodt, D. Goltzsche, C. Fetzer, P. Pietzuch, and R. Kapitza. SecureKeeper: Confidential ZooKeeper using Intel SGX. In Int. Middleware Conference. ACM, 2016.

[9] E. Brickell and J. Li. Enhanced privacy id from bilinear pairing for hardware authentication and attestation. Int. Journal of Information Privacy, Security and Integrity 2, 2011.

[10] C. Cachin, I. Keidar, and A. Shraer. Fail-aware untrusted storage. SIAM Journal on Computing, 2011.

[11] C. Cachin and O. Ohrimenko. Verifying the consistency of remote untrusted services with commutative operations. In Conference on Principles of Distributed Systems (OPODIS). Springer, 2014.

[12] C. Cachin, A. Shelat, and A. Shraer. Efficient fork-linearizable access to untrusted shared memory. In Proc. on Principles of Distributed Computing (PODC). ACM, 2007.

[13] B.-G. Chun, C. Curino, R. Sears, A. Shraer, S. Madden, and R. Ramakrishnan. Mobius: Unified messaging and data serving for mobile apps. In Intl. Conference on Mobile Systems, Applications, and Services (MobiSys). ACM, 2012.

[14] B.-G. Chun, P. Maniatis, S. Shenker, and J. Kubiatowicz. Attested append-only memory: Making adversaries stick to their word. In SIGOPS Operating Systems Review. ACM, 2007.

[15] B.-G. Chun, P. Maniatis, S. Shenker, and J. Kubiatowicz. Attested append-only memory: Making adversaries stick to their word. In Proc. on Operating Systems Principles (SOSP). ACM, 2007.

[16] B. F. Cooper, A. Silberstein, E. Tam, R. Ramakrishnan, and R. Sears. Benchmarking cloud serving systems with YCSB. In Proc. on Cloud Computing (SoCC). ACM, 2010.

[17] A. J. Feldman, W. P. Zeller, M. J. Freedman, and E. W. Felten. SPORC: Group collaboration using untrusted cloud resources. In Proc. Operating Systems Design and Implementation (OSDI), 2010.

[18] M. T. Goodrich, C. Papamanthou, R. Tamassia, and N. Triandopoulos. Athos: Efficient authentication of outsourced file systems. In Intl. Conference on Information Security (ISC). Springer, 2008.

[19] S. Gueron. A memory encryption engine suitable for general purpose processors. Cryptology ePrint Archive, Report 2016/204, 2016.

[20] M. P. Herlihy and J. M. Wing. Linearizability: A correctness condition for concurrent objects. Transactions on Programming Languages and Systems, 1990.

[21] M. Hoekstra, R. Lal, P. Pappachan, V. Phegade, and J. Del Cuvillo. Using innovative instructions to create trustworthy software solutions. In Int. Workshop on Hardware and Architectural Support for Security and Privacy (HASP), 2013.

[22] Intel. Intel SGX SDK Developer Reference for Windows* OS, 2015. https://software. intel.com/en-us/sgx-sdk/documentation. 
[23] Intel. Intel SGX SDK for Linux* OS, 2016. https://01. org/intel-software-guard-extensions/documentation/ intel-sgx-sdk-developer-reference.

[24] K. R. Jayaram, D. Safford, U. Sharma, V. Naik, D. Pendarakis, and S. Tao. Trustworthy geographically fenced hybrid clouds. In Int. Middleware Conference, 2014.

[25] B. Laurie. Certificate transparency. Communications of the ACM, 2014.

[26] D. Levin, J. R. Douceur, J. R. Lorch, and T. Moscibroda. TrInc: Small trusted hardware for large distributed systems. In Proc. Networked Systems Design and Implementation (NSDI), 2009.

[27] J. Li, M. Krohn, D. Mazières, and D. Shasha. Secure untrusted data repository (SUNDR). In Symp. Operating Systems Design and Implementation (OSDI), 2004.

[28] M. Majuntke, D. Dobre, M. Serafini, and N. Suri. Abortable fork-linearizable storage. In Conference on Principles of Distributed Systems (OPODIS). Springer, 2009.

[29] S. Matetic, M. Ahmed, K. Kostiainen, A. Dhar, D. Sommer, A. Gervais, A. Juels, and S. Capkun. Rote: Rollback protection for trusted execution. Cryptology ePrint Archive, Report 2017/048, 2017.

[30] D. Mazières and D. Shasha. Building secure file systems out of byzantine storage. In Proc. on Principles of Distributed Computing (PODC). ACM, 2002.

[31] F. McKeen, I. Alexandrovich, A. Berenzon, C. V. Rozas, H. Shafi, V. Shanbhogue, and U. R. Savagaonkar. Innovative instructions and software model for isolated execution. In Int. Workshop on Hardware and Architectural Support for Security and Privacy (HASP). ACM, 2013.

[32] B. Parno, J. R. Lorch, J. R. Douceur, J. Mickens, and J. M. McCune. Memoir: Practical state continuity for protected modules. In IEEE Proc. on Security \& Privacy, 2011.

[33] S. Pearson and A. Benameur. Privacy, security and trust issues arising from cloud computing. In Proc. Cloud Computing (CloudCom), 2010.

[34] F. Schuster, M. Costa, C. Fournet, C. Gkantsidis, M. Peinado, G. Mainar-Ruiz, and M. Russinovich. VC3: trustworthy data analytics in the cloud using SGX. In IEEE Proc. on Security \& Privacy, 2015.

[35] A. Shraer, C. Cachin, A. Cidon, I. Keidar, Y. Michalevsky, and D. Shaket. Venus: Verification for untrusted cloud storage. In Proc. Cloud Computing Security Workshop (CCSW). ACM, 2010.

[36] R. Strackx, B. Jacobs, and F. Piessens. ICE: A passive, high-speed, state-continuity scheme. In Annual Computer Security Applications Conference. ACM, 2014.

[37] R. Strackx and N. Lambrigts. Idea: State-continuous transfer of state in protected-module architectures. In Engineering Secure Software and Systems. Springer, 2015.

[38] R. Strackx and F. Piessens. Ariadne: A minimal approach to state continuity. In USENIX Security Symposium, 2016.

[39] D. B. Terry, M. Theimer, K. Petersen, A. J. Demers, M. Spreitzer, and C. Hauser. Managing update conflicts in Bayou, a weakly connected replicated storage system. In ACM Symposium on Operating System Principles (SOSP), 1995. 
[40] P. Williams, R. Sion, and D. Shasha. The blind stone tablet: Outsourcing durability to untrusted parties. In Proc. Network and Distributed Systems Security Symposium (NDSS), 2009. 